Теорія Ймовір. та Матем. Статист. Вип. 78, 2008
Theor. Probability and Math. Statist. No. 78, 2009, Pages 115-131 S 0094-9000(09)00766-2

Article electronically published on August 4, 2009

\title{
CONVERGENCE OF A SEQUENCE OF MARKOV CHAINS TO A DIFFUSION TYPE PROCESS
}

UDC 519.21

\author{
G. L. KULINICH AND A. V. YERSHOV
}

\begin{abstract}
A random polygonal line constructed from a sequence of series of homogeneous Markov chains is considered under rather nonregular dependence of their local characteristics on a series number. Sufficient conditions are obtained for the weak convergence of a random polygonal line to a diffusion type process. The conditions are expressed explicitly in terms of local characteristics of the Markov chains.
\end{abstract}

\section{INTRODUCTION}

Let $\xi_{n 0}, \xi_{n 1}, \ldots, \xi_{n k_{n}}$ be a sequence of random variables that form a Markov chain for each fixed $n$ and let $0=t_{n 0}<t_{n 1}<\cdots<t_{n k_{n}}=1$ be a partition of the interval $[0,1]$ such that $\lambda_{n}=\max _{k}\left[t_{n k+1}-t_{n k}\right]$ approaches zero as $n \rightarrow \infty$. Put $\Delta t_{n k}=t_{n k+1}-t_{n k}$, $\Delta \xi_{n k}=\xi_{n k+1}-\xi_{n k}$, and let $\mathcal{F}_{n k}$ be the minimal $\sigma$-algebra generated by the random variables $\xi_{n 0}, \xi_{n 1}, \ldots, \xi_{n k}$,

$$
\begin{gathered}
a_{n}(x)=\frac{1}{\Delta t_{n k}} \mathrm{E}\left\{\Delta \xi_{n k} \mid \xi_{n k}=x\right\}, \\
\sigma_{n}^{2}(x)=\frac{1}{\Delta t_{n k}} \mathrm{E}\left\{\left[\Delta \xi_{n k}\right]^{2} \mid \xi_{n k}=x\right\}-a_{n}^{2}(x) \Delta t_{n k}, \\
\Delta \omega_{n k}=\left[\Delta \xi_{n k}-a_{n}\left(\xi_{n k}\right) \Delta t_{n k}\right] \sigma_{n}^{-1}\left(\xi_{n k}\right) .
\end{gathered}
$$

Let $\zeta_{n}(t)$ be a random polygonal line with vertices at the points $\left(t_{n k}, \zeta_{n k}\right), \zeta_{n k}=G_{n}\left(\xi_{n k}\right)$, where $G_{n}(x)$ is some family of unbounded twice continuously differentiable functions, $x \in \mathbb{R}^{1}$, and

$$
w_{n}(t)=\sum_{t_{n k}<t} \Delta \omega_{n k} .
$$

There are many publications devoted to limit theorems for Markov sequences and for functionals of Markov chains. We mention the book [1] (Chapter IX, §4) where the weak convergence of a random polygonal line with vertices at the points $\left(t_{n k}, \xi_{n k}\right)$ to a diffusion process is considered under the assumption that the functions $a_{n}(x) \sigma_{n}^{-1}(x)$ and $\sigma_{n}^{-1}(x)$ are uniformly bounded in $n$, and the paper [4] where the same question is studied under the assumption that the functions $a_{n}(x)$ and $\sigma_{n}(x)$ can be unbounded at some points $x_{k}$. In the paper [4,

$$
G_{n}(x)=\int_{0}^{x} \exp \left\{-2 \int_{0}^{u} \frac{a_{n}(v)}{\sigma_{n}^{2}(v)} d v\right\} d u .
$$

2000 Mathematics Subject Classification. Primary 60H10; Secondary 34G10, 47A50, 47D06.

Key words and phrases. A sequence of series of Markov chains, nonregular dependence of local characteristics of Markov chains on the number of a series, a random polygonal line, weak convergence, stochastic differential equation, diffusion type processes. 
Another problem studied in [4] is the weak convergence of a random polygonal line with vertices at the points $\left(t_{n k}, \sum_{i=0}^{k} g_{n}\left(\xi_{n i}\right) \Delta t_{n i}\right)$ to a certain functional of the limit process for $\zeta_{n}(t)$.

Sufficient conditions for the compactness of finite-dimensional distributions of the process $\zeta_{n}(t)$ (Theorem 2.1) and for the weak convergence of this process to a diffusion type process (Theorems 3.1 and 3.2) are obtained in this paper.

Similar results for solutions of stochastic differential equations under rather irregular dependence of the coefficients on the parameter are obtained in the papers [5, 6].

In what follows we use the following notation:

$$
\begin{gathered}
L G_{n}(x)=G_{n}^{\prime}(x) a_{n}(x)+\frac{1}{2} G_{n}^{\prime \prime}(x) \sigma_{n}^{2}(x), \\
\eta_{i}=\int_{\xi_{n i}}^{\xi_{n i+1}} \int_{\xi_{n i}}^{x}\left[G_{n}^{\prime \prime}(u)-G_{n}^{\prime \prime}\left(\xi_{n i}\right)\right] d u d x, \\
P_{N}=\mathrm{P}\left\{\sup _{0 \leq t \leq 1}\left|\zeta_{n}(t)\right|>N\right\}, \quad A^{(\varepsilon)}=\bigcup_{k=1}^{m}\left\{x:\left|x-x_{k}\right|<\varepsilon\right\} .
\end{gathered}
$$

The convergence in probability as $n \rightarrow \infty$ is denoted by $\xi_{n} \stackrel{P}{\rightarrow} \xi$. Let $\chi_{A}(x)$ be the indicator of a set $A$. The symbols $K$ and $C$ with or without subscripts denote the constants whose values do not matter for the purposes of this paper.

\section{The compactness}

Assume that there exists a family of twice continuously differentiable functions $G_{n}(x)$, $x \in \mathbb{R}^{1}$, such that

$\left(I_{1}\right)$ for some constant $K>0$, the following inequality holds:

$$
\left[L G_{n}(x)\right]^{2}+\left[G_{n}^{\prime}(x) \sigma_{n}(x)\right]^{2} \leq K\left(1+\left[G_{n}(x)\right]^{2}\right)
$$

for all $x$ and $n$,

(I $I_{2} \lim _{n \rightarrow \infty} \mathrm{E}\left(\sum_{i=0}^{k_{n}-1}\left|\eta_{i}\right|\right)^{2}=0$

$\left(I_{3}\right) \lim _{n \rightarrow \infty} \sum_{i=0}^{k_{n}-1} \mathrm{E}\left|G_{n}^{\prime \prime}\left(\xi_{n i}\right)\right|^{2}\left|\Delta \xi_{n i}\right|^{4}=0$,

$\left(I_{4}\right) \lim _{n \rightarrow \infty} \lambda_{n} \sup _{x}\left|G_{n}^{\prime \prime}(x)\right|\left[a_{n}^{2}(x)+\left|a_{n}(x) \sigma_{n}(x)\right|\right]=0$,

$\left(I_{5}\right) \lim _{n \rightarrow \infty} \lambda_{n} \sup _{x}\left|G_{n}^{\prime \prime}(x) \sigma_{n}^{2}(x)\right|^{2}=0$,

$\left(I_{6}\right) \mathrm{E}\left|G_{n}\left(\xi_{n 0}\right)\right|^{2} \leq C$,

$\left(I_{7}\right)$

$$
\lim _{n \rightarrow \infty} \sum_{i=0}^{k_{n}-1} \mathrm{E}\left|\frac{\Delta \xi_{n i}}{\sigma_{n}\left(\xi_{n i}\right)}\right|^{4}=0, \quad \lim _{n \rightarrow \infty} \lambda_{n}^{3} \lim _{n \rightarrow \infty} \sup _{x}\left|\frac{a_{n}(x)}{\sigma_{n}(x)}\right|^{4}=0 .
$$

Theorem 2.1. Assume that conditions $\left(I_{1}\right)-\left(I_{6}\right)$ hold. Then there are a subsequence $n^{\prime} \rightarrow \infty$, a probability space $(\tilde{\Omega}, \tilde{\mathcal{F}}, \tilde{P})$, and stochastic processes $\tilde{\zeta}_{n^{\prime}}(t)$ and $\tilde{\zeta}_{0}(t)$ defined on this space such that all finite-dimensional distributions of the process $\tilde{\zeta}_{n^{\prime}}(t)$ coincide with the corresponding finite-dimensional distributions of the process $\zeta_{n^{\prime}}(t)$. Moreover, $\tilde{\zeta}_{n^{\prime}}(t) \stackrel{\tilde{\mathrm{P}}}{\rightarrow} \tilde{\zeta}_{0}(t)$ as $n^{\prime} \rightarrow \infty$ for all $t \geq 0$.

Proof. It is clear that

$$
\zeta_{n k+1}=\zeta_{n 0}+\sum_{i=0}^{k}\left[\zeta_{n i+1}-\zeta_{n i}\right]=\zeta_{n 0}+\alpha_{n k+1}+\gamma_{n k+1}+\beta_{n k+1},
$$


where

$$
\begin{gathered}
\alpha_{n k}=\sum_{i=0}^{k-1} L G_{n}\left(\xi_{n i}\right) \Delta t_{n i}, \quad \gamma_{n k}=\sum_{i=0}^{k-1} G_{n}^{\prime}\left(\xi_{n i}\right) \sigma_{n}\left(\xi_{n i}\right) \Delta \omega_{n i}, \\
\beta_{n k}=\frac{1}{2} \sum_{i=0}^{k-1} G_{n}^{\prime \prime}\left(\xi_{n i}\right)\left[a_{n}^{2}\left(\xi_{n i}\right)\left(\Delta t_{n i}\right)^{2}\right. \\
\left.\quad+2 a_{n}\left(\xi_{n i}\right) \sigma_{n}\left(\xi_{n i}\right) \Delta t_{n i} \Delta \omega_{n i}+\sigma_{n}^{2}\left(\xi_{n i}\right)\left[\left(\Delta \omega_{n i}\right)^{2}-\Delta t_{n i}\right]\right] \\
+\sum_{i=0}^{k-1} \eta_{i} .
\end{gathered}
$$

Let

$$
\chi_{n k}^{(N)}=\chi_{|x| \leq N}\left(\sup _{0 \leq i \leq k}\left|\zeta_{n i}\right|\right) .
$$

Since $\chi_{n k}^{(N)}=\chi_{n k}^{(N)} \cdot \chi_{n i}^{(N)}$ with probability one for $i \leq k$, we obtain from equality (1) that

$$
\zeta_{n k+1} \chi_{n k+1}^{(N)}=\zeta_{n 0} \chi_{n k+1}^{(N)}+\alpha_{n k+1}^{(N)} \chi_{n k+1}^{(N)}+\gamma_{n k+1}^{(N)} \chi_{n k+1}^{(N)}+\beta_{n k+1}^{(N)} \chi_{n k+1}^{(N)},
$$

where

$$
\begin{aligned}
& \alpha_{n k}^{(N)}=\sum_{i=0}^{k-1} L G_{n}\left(\xi_{n i}\right) \chi_{n i}^{(N)} \Delta t_{n i}, \quad \gamma_{n k}^{(N)}=\sum_{i=0}^{k-1} G_{n}^{\prime}\left(\xi_{n i}\right) \sigma_{n}\left(\xi_{n i}\right) \chi_{n i}^{(N)} \Delta \omega_{n i}, \\
& \beta_{n k}^{(N)}=\frac{1}{2} \sum_{i=0}^{k-1} G_{n}^{\prime \prime}\left(\xi_{n i}\right)[ a_{n}^{2}\left(\xi_{n i}\right)\left(\Delta t_{n i}\right)^{2} \\
&\left.+2 a_{n}\left(\xi_{n i}\right) \sigma_{n}\left(\xi_{n i}\right) \Delta t_{n i} \Delta \omega_{n i}+\sigma_{n}^{2}\left(\xi_{n i}\right)\left(\Delta \omega_{n i}\right)^{2}-\Delta t_{n i}\right] \chi_{n i}^{(N)} \\
&+ \sum_{i=0}^{k-1} \eta_{i} \chi_{n i}^{(N)} \chi_{n i+1}^{(N)} .
\end{aligned}
$$

We square both sides of equality (2), then use the inequality

$$
\left(\sum_{i=1}^{N} a_{i}\right)^{2} \leq N \sum_{i=1}^{N} a_{i}^{2}
$$

and take the mathematical expectations. Thus we get

$$
\mathrm{E} \zeta_{n k+1}^{2} \chi_{n k+1}^{(N)} \leq 4\left(\mathrm{E} \zeta_{n 0}^{2}+\mathrm{E}\left[\alpha_{n k+1}^{(N)}\right]^{2}+\mathrm{E}\left[\gamma_{n k+1}^{(N)}\right]^{2}+\mathrm{E}\left[\beta_{n k+1}^{(N)}\right]^{2}\right)
$$

The terms on the right hand side of inequality (3) can be estimated according to the assumptions of the theorem. Namely,

$$
\begin{gathered}
\mathrm{E} \zeta_{n 0}^{2} \leq C, \\
\mathrm{E}\left[\alpha_{n k+1}^{(N)}\right]^{2} \leq \mathrm{E} \sum_{i=0}^{k} \Delta t_{n i} \sum_{i=0}^{k} K\left(1+\zeta_{n i}^{2} \chi_{n i}^{(N)}\right) \Delta t_{n i} \leq \sum_{i=0}^{k} K\left(1+\mathrm{E} \zeta_{n i}^{2} \chi_{n i}^{(N)}\right) \Delta t_{n i}, \\
\mathrm{E}\left[\gamma_{n k+1}^{(N)}\right]^{2}=\sum_{i=0}^{k} \mathrm{E}\left[G_{n}^{\prime}\left(\xi_{n i}\right) \sigma_{n}\left(\xi_{n i}\right)\right]^{2} \chi_{n i}^{(N)} \Delta t_{n i} \leq \sum_{i=0}^{k} K\left(1+\mathrm{E} \zeta_{n i}^{2} \chi_{n i}^{(N)}\right) \Delta t_{n i} .
\end{gathered}
$$


Here we used the martingale property of the sequence $\gamma_{n k}^{(N)}$ with respect to $k$. Further,

$$
\begin{aligned}
& \mathrm{E} \sup _{0 \leq k \leq k_{n}}\left|\beta_{n k}^{(N)}\right|^{2} \\
& \leq 4\left\{\operatorname{Esup}_{k}\left[\frac{1}{2} \sum_{i=0}^{k-1} G_{n}^{\prime \prime}\left(\xi_{n i}\right) a_{n}^{2}\left(\xi_{n i}\right) \chi_{n i}^{(N)}\left(\Delta t_{n i}\right)^{2}\right]^{2}\right. \\
& +\mathrm{E} \sup _{k}\left[\sum_{i=0}^{k-1} G_{n}^{\prime \prime}\left(\xi_{n i}\right) a_{n}\left(\xi_{n i}\right) \sigma_{n}\left(\xi_{n i}\right) \chi_{n i}^{(N)} \Delta t_{n i} \Delta \omega_{n i}\right]^{2} \\
& +\mathrm{E} \sup _{k}\left[\frac{1}{2} \sum_{i=0}^{k-1} G_{n}^{\prime \prime}\left(\xi_{n i}\right) \sigma_{n}^{2}\left(\xi_{n i}\right) \chi_{n i}^{(N)}\left[\left(\Delta \omega_{n i}\right)^{2}-\Delta t_{n i}\right]\right]^{2} \\
& \left.+\mathrm{E} \sup _{k}\left[\sum_{i=0}^{k-1} \eta_{i} \chi_{n i}^{(N)} \chi_{n i+1}^{(N)}\right]^{2}\right\} \\
& \leq 4\left\{\frac{1}{4} \sup _{x}\left|G_{n}^{\prime \prime}(x) a_{n}^{2}(x)\right|^{2} \cdot \lambda_{n}^{2}\right. \\
& +4 \mathrm{E}\left(\sum_{i=0}^{k_{n}-1} G_{n}^{\prime \prime}\left(\xi_{n i}\right) a_{n}\left(\xi_{n i}\right) \sigma_{n}\left(\xi_{n i}\right) \chi_{n i}^{(N)} \Delta t_{n i} \Delta \omega_{n i}\right)^{2} \\
& +\mathrm{E}\left(\sum_{i=0}^{k_{n}-1} G_{n}^{\prime \prime}\left(\xi_{n i}\right) \sigma_{n}^{2}\left(\xi_{n i}\right) \chi_{n i}^{(N)}\left[\left(\Delta \omega_{n i}\right)^{2}-\Delta t_{n i}\right]\right)^{2} \\
& \left.+\mathrm{E}\left(\sum_{i=0}^{k_{n}-1}\left|\eta_{i}\right|\right)^{2}\right\} \\
& \leq 4\left\{\frac{1}{4} \lambda_{n}^{2} \sup _{x}\left|G_{n}^{\prime \prime}(x) a_{n}^{2}(x)\right|^{2}\right. \\
& +4 \sum_{i=0}^{k_{n}-1} \mathrm{E}\left[G_{n}^{\prime \prime}\left(\xi_{n i}\right) a_{n}\left(\xi_{n i}\right) \sigma_{n}\left(\xi_{n i}\right)\right]^{2} \chi_{n i}^{(N)}\left(\Delta t_{n i}\right)^{3} \\
& \left.+\sum_{i=0}^{k_{n}-1} \mathrm{E}\left[G_{n}^{\prime \prime}\left(\xi_{n i}\right) \sigma_{n}^{2}\left(\xi_{n i}\right)\right]^{2} \chi_{n i}^{(N)}\left[\left(\Delta \omega_{n i}\right)^{2}-\Delta t_{n i}\right]^{2}+c_{n}\right\} \\
& \leq 4\left\{\frac{1}{4} \lambda_{n}^{2} \sup _{x}\left|G_{n}^{\prime \prime}(x) a_{n}^{2}(x)\right|^{2}\right. \\
& +4 \lambda_{n}^{2} \sup _{x}\left|G_{n}^{\prime \prime}(x) a_{n}(x) \sigma_{n}(x)\right|^{2}+16 \sum_{i=0}^{k_{n}-1} \mathrm{E}\left[G_{n}^{\prime \prime}\left(\xi_{n i}\right)\right]^{2}\left(\Delta \xi_{n i}\right)^{4} \\
& +16 \sum_{i=0}^{k_{n}-1} \mathrm{E}\left[G_{n}^{\prime \prime}\left(\xi_{n i}\right)\right]^{2}\left(a_{n}\left(\xi_{n i}\right) \Delta t_{n i}\right)^{4} \\
& \left.+2 \sum_{i=0}^{k_{n}-1} \mathrm{E}\left[G_{n}^{\prime \prime}\left(\xi_{n i}\right) \sigma_{n}^{2}\left(\xi_{n i}\right)\right]^{2}\left(\Delta t_{n i}\right)^{2}+c_{n}\right\}
\end{aligned}
$$




$$
\begin{aligned}
& \leq 4\left\{\frac{1}{4} \lambda_{n}^{2} \sup _{x}\left|G_{n}^{\prime \prime}(x) a_{n}^{2}(x)\right|^{2}+4 \lambda_{n}^{2} \sup _{x}\left|G_{n}^{\prime \prime}(x) a_{n}(x) \sigma_{n}(x)\right|^{2}\right. \\
& +16 \sum_{i=0}^{k_{n}-1} \mathrm{E}\left[G_{n}^{\prime \prime}\left(\xi_{n i}\right)\right]^{2}\left(\Delta \xi_{n i}\right)^{4}+16 \lambda_{n}^{3} \sup _{x}\left(G_{n}^{\prime \prime}(x) a_{n}^{2}(x)\right)^{2} \\
& \left.+2 \lambda_{n} \sup _{x}\left|G_{n}^{\prime \prime}(x) \sigma_{n}^{2}(x)\right|^{2}+c_{n}\right\} \\
& =\hat{c}_{n},
\end{aligned}
$$

where $\hat{c}_{n}$ is a sequence that does not depend on $N$ and such that $\hat{c}_{n} \rightarrow 0$ as $n \rightarrow \infty$. We again used the martingale property in $k$ of both the second and third terms in the expression for $\beta_{n k}^{(N)}$.

Relations (3) -(6) imply that there are constants $C_{1}$ and $K_{1}$ that do not depend on $n$ and $N$ and such that

$$
b_{n k+1}^{(N)} \leq C_{1}+K_{1} \sum_{i=0}^{k} b_{n i}^{(N)} \Delta t_{n i}
$$

for all $0<k<k_{n}-1$ and $n$, where $b_{n k}^{(N)}=\mathrm{E} \zeta_{n k}^{2} \chi_{n k}^{(N)}$. The Gronwall-Bellman inequality yields the bound $b_{n k+1}^{(N)} \leq C_{1} \exp \left\{\sum_{i=0}^{k} K_{1} \Delta t_{n i}\right\} \leq C^{*}$, where $C^{*}=C_{1} e^{K_{1}}$. Passing to the limit as $N \uparrow \infty$ we obtain the upper estimate $\sup _{0 \leq k \leq k_{n}} \mathrm{E} \zeta_{n k}^{2} \leq C^{*}$. Moreover,

$$
\mathrm{E} \sup _{0 \leq k \leq k_{n}}\left|\beta_{n k}\right|^{2} \leq \hat{c}_{n}
$$

by (6), where $\hat{c}_{n} \rightarrow 0$ as $n \rightarrow \infty$.

Analogous inequalities (4) and (5) imply

$$
\begin{aligned}
& \mathrm{E} \sup _{0 \leq k \leq k_{n}}\left|\alpha_{n k}\right|^{2} \leq \sum_{i=0}^{k_{n}-1} K\left(1+\mathrm{E} \zeta_{n i}^{2}\right) \Delta t_{n i}, \\
& \mathrm{E} \sup _{0 \leq k \leq k_{n}}\left|\gamma_{n k}\right|^{2} \leq \sum_{i=0}^{k_{n}-1} K\left(1+\mathrm{E} \zeta_{n i}^{2}\right) \Delta t_{n i} .
\end{aligned}
$$

Thus

$$
\mathrm{E} \sup _{0 \leq k \leq k_{n}}\left|\zeta_{n k}\right|^{2} \leq \hat{C} .
$$

Consider the random polygonal line with vertices at the points $\left(t_{n k}, \zeta_{n k}\right)$, that is,

$$
\zeta_{n}(t)=\zeta_{n k}+\frac{t-t_{n k}}{\Delta t_{n k}} \Delta \zeta_{n k}
$$

for $t_{n k} \leq t \leq t_{n k+1}$, where $\Delta \zeta_{n k}=\zeta_{n k+1}-\zeta_{n k}$. We also consider the random polygonal lines $\alpha_{n}(t), \gamma_{n}(t)$, and $\beta_{n}(t)$ with vertices at the points $\left(t_{n k}, \alpha_{n k}\right),\left(t_{n k}, \gamma_{n k}\right)$, and $\left(t_{n k}, \beta_{n k}\right)$, respectively. Then

$$
\zeta_{n}(t)=\zeta_{n}(0)+\alpha_{n}(t)+\gamma_{n}(t)+\beta_{n}(t)
$$

with probability one for all $t \in[0,1]$. 
According to definition (9), $\left|\zeta_{n}(t)\right| \leq 2\left|\zeta_{n k}\right|+\left|\zeta_{n k+1}\right|$ for $t_{n k} \leq t \leq t_{n k+1}$. Thus

$$
\begin{gathered}
\sup _{0 \leq t \leq 1} \mathrm{P}\left\{\left|\zeta_{n}(t)\right|>C\right\} \leq \sup _{k} \mathrm{P}\left\{2\left|\zeta_{n k}\right|+\left|\zeta_{n k+1}\right|>C\right\} \\
\leq \sup _{k} \mathrm{P}\left\{\left|\zeta_{n k}\right|>\frac{C}{4}\right\}+\sup _{k} \mathrm{P}\left\{\left|\zeta_{n k+1}\right|>\frac{C}{2}\right\} \\
\leq\left[\left(\frac{4}{C}\right)^{2}+\left(\frac{2}{C}\right)^{2}\right] \sup _{k} \mathrm{E} \zeta_{n k}^{2} \leq \frac{20}{C^{2}} \cdot C^{*} .
\end{gathered}
$$

Hence

$$
\lim _{C \rightarrow \infty} \varlimsup_{n \rightarrow \infty} \sup _{0 \leq t \leq 1} \mathrm{P}\left\{\left|\zeta_{n}(t)\right|>C\right\}=0 .
$$

Let $0 \leq t^{\prime}<t^{\prime \prime} \leq 1$ for $t^{\prime} \in\left[t_{n i-1}, t_{n i}\right]$ and $t^{\prime \prime} \in\left[t_{n k}, t_{n k+1}\right]$. Then we obtain from definition (9) that

$\left|\zeta_{n}\left(t^{\prime \prime}\right)-\zeta_{n}\left(t^{\prime}\right)\right| \leq\left|\zeta_{n}\left(t_{n k}\right)-\zeta_{n}\left(t_{n i}\right)\right|+\left|\zeta_{n}\left(t_{n k+1}\right)-\zeta_{n}\left(t_{n k}\right)\right|+\left|\zeta_{n}\left(t_{n i}\right)-\zeta_{n}\left(t_{n i-1}\right)\right|$, whence

$$
\begin{aligned}
\sup _{\left|t^{\prime}-t^{\prime \prime}\right| \leq h} \mathrm{P}\left\{\left|\zeta_{n}\left(t^{\prime \prime}\right)-\zeta_{n}\left(t^{\prime}\right)\right|>\varepsilon\right\} \leq & \sup _{\left|t_{n k}-t_{n i}\right| \leq h} \mathrm{P}\left\{\left|\zeta_{n}\left(t_{n k}\right)-\zeta_{n}\left(t_{n i}\right)\right|>\frac{\varepsilon}{3}\right\} \\
& +2 \sup _{k} \mathrm{P}\left\{\left|\zeta_{n}\left(t_{n k+1}\right)-\zeta_{n}\left(t_{n k}\right)\right|>\frac{\varepsilon}{3}\right\} .
\end{aligned}
$$

An analogous inequality holds for each of the processes $\alpha_{n}(t), \gamma_{n}(t)$, and $\beta_{n}(t)$. We estimate the corresponding probabilities further for each of these processes separately. Since

$$
\begin{gathered}
\sup _{\left|t_{n k}-t_{n i}\right| \leq h} \mathrm{P}\left\{\left|\alpha_{n}\left(t_{n k}\right)-\alpha_{n}\left(t_{n i}\right)\right|>\varepsilon\right\} \leq \sup _{\left|t_{n k}-t_{n i}\right| \leq h} \frac{1}{\varepsilon^{2}} \mathrm{E}\left(\sum_{j=i}^{k-1} L G_{n}\left(\xi_{n j}\right) \Delta t_{n j}\right)^{2} \\
\leq \frac{1}{\varepsilon^{2}} \sup _{\left|t_{n k}-t_{n i}\right| \leq h} \sum_{j=i}^{k-1} \Delta t_{n j} \sum_{j=i}^{k-1} K(1+\hat{C}) \Delta t_{n j} \leq \frac{1}{\varepsilon^{2}} K(1+\hat{C}) h^{2}, \\
\sup _{k} \mathrm{P}\left\{\left|\alpha_{n}\left(t_{n k+1}\right)-\alpha_{n}\left(t_{n k}\right)\right|>\varepsilon\right\} \leq \frac{1}{\varepsilon^{2}} \sup _{k} \mathrm{E}\left(L G_{n}\left(\xi_{n k}\right) \Delta t_{n k}\right)^{2} \leq \frac{1}{\varepsilon^{2}} K(1+\hat{C}) \lambda_{n}^{2},
\end{gathered}
$$

we have

$$
\lim _{h \rightarrow 0} \varlimsup_{n \rightarrow \infty} \sup _{\left|t^{\prime}-t^{\prime \prime}\right| \leq h} \mathrm{P}\left\{\left|\alpha_{n}\left(t^{\prime \prime}\right)-\alpha_{n}\left(t^{\prime}\right)\right|>\varepsilon\right\}=0 .
$$

Since $\gamma_{n k}$ is a martingale, we derive the following bounds:

$$
\begin{gathered}
\sup _{\left|t_{n k}-t_{n i}\right| \leq h} \mathrm{P}\left\{\left|\gamma_{n}\left(t_{n k}\right)-\gamma_{n}\left(t_{n i}\right)\right|>\varepsilon\right\} \leq \frac{1}{\varepsilon^{2}} \sup _{\left|t_{n k}-t_{n i}\right| \leq h} \mathrm{E}\left(\sum_{j=i}^{k-1} G_{n}^{\prime}\left(\xi_{n j}\right) \sigma_{n}\left(\xi_{n j}\right) \Delta w_{n j}\right)^{2} \\
=\frac{1}{\varepsilon^{2}} \sup _{\left|t_{n k}-t_{n i}\right| \leq h} \sum_{j=i}^{k-1} \mathrm{E}\left[G_{n}^{\prime}\left(\xi_{n j}\right) \sigma_{n}\left(\xi_{n j}\right)\right]^{2} \Delta t_{n j} \leq \frac{1}{\varepsilon^{2}} K(1+\hat{C}) h, \\
\sup _{k} \mathrm{P}\left\{\left|\gamma_{n}\left(t_{n k+1}\right)-\gamma_{n}\left(t_{n k}\right)\right|>\varepsilon\right\} \leq \frac{1}{\varepsilon^{2}} \sup _{k} \mathrm{E}\left[G_{n}^{\prime}\left(\xi_{n k}\right) \sigma_{n}\left(\xi_{n k}\right) \Delta \omega_{n k}\right]^{2} \\
=\frac{1}{\varepsilon^{2}} \sup _{k} \mathrm{E}\left[G_{n}^{\prime}\left(\xi_{n k}\right) \sigma_{n}\left(\xi_{n k}\right)\right]^{2} \Delta t_{n k} \leq \frac{1}{\varepsilon^{2}} K(1+\hat{C}) \cdot \lambda_{n} .
\end{gathered}
$$

Thus

$$
\lim _{h \rightarrow 0} \varlimsup_{n \rightarrow \infty} \sup _{\left|t^{\prime}-t^{\prime \prime}\right| \leq h} \mathrm{P}\left\{\left|\gamma_{n}\left(t^{\prime \prime}\right)-\gamma_{n}\left(t^{\prime}\right)\right|>\varepsilon\right\}=0
$$


Moreover

$$
\begin{aligned}
\lim _{h \rightarrow 0} & \lim _{n \rightarrow \infty} \sup _{\left|t^{\prime}-t^{\prime \prime}\right| \leq h} \mathrm{P}\left\{\left|\beta_{n}\left(t^{\prime \prime}\right)-\beta_{n}\left(t^{\prime}\right)\right|>\varepsilon\right\} \\
& \leq \lim _{h \rightarrow 0} \varlimsup_{n \rightarrow \infty} \sup _{\left|t^{\prime}-t^{\prime \prime}\right| \leq h}\left[\mathrm{P}\left\{\left|\beta_{n}\left(t^{\prime \prime}\right)\right|>\frac{\varepsilon}{2}\right\}+\mathrm{P}\left\{\left|\beta_{n}\left(t^{\prime}\right)\right|>\frac{\varepsilon}{2}\right\}\right] \\
& \leq 2\left(\frac{2}{\varepsilon}\right)^{2} \lim _{h \rightarrow 0} \varlimsup_{n \rightarrow \infty} \sup _{0 \leq t \leq 1} \mathrm{E}\left|\beta_{n}(t)\right|^{2} \leq 18\left(\frac{2}{\varepsilon}\right)^{2} \varlimsup_{n \rightarrow \infty} \sup _{k} \mathrm{E}\left|\beta_{n k}\right|^{2}=0 .
\end{aligned}
$$

Taking into account (10) and (13)-(15) we get

$$
\lim _{h \rightarrow 0} \varlimsup_{n \rightarrow \infty} \sup _{\left|t^{\prime}-t^{\prime \prime}\right| \leq h} \mathrm{P}\left\{\left|\zeta_{n}\left(t^{\prime \prime}\right)-\zeta_{n}\left(t^{\prime}\right)\right|>\varepsilon\right\}=0 .
$$

Equalities (12) and (16) complete the proof of Theorem 2.1 (see [8, $\S 1$ ).

\section{The CONVERGENCE TO DIFFUSION TYPE PROCESSES}

It is clear that relationships similar to (11) hold for the processes $\alpha_{n}(t), \gamma_{n}(t)$, and $\beta_{n}(t)$, too. Thus we obtain

$$
\lim _{C \rightarrow \infty} \varlimsup_{n \rightarrow \infty} \sup _{0 \leq t \leq 1} \mathrm{P}\left\{\left|\alpha_{n}(t)\right|>C\right\}=0
$$

by (4),

$$
\lim _{C \rightarrow \infty} \varlimsup_{n \rightarrow \infty} \sup _{0 \leq t \leq 1} \mathrm{P}\left\{\left|\gamma_{n}(t)\right|>C\right\}=0
$$

by (5), and

$$
\lim _{C \rightarrow \infty} \varlimsup_{n \rightarrow \infty} \sup _{0 \leq t \leq 1} \mathrm{P}\left\{\left|\beta_{n}(t)\right|>C\right\}=0
$$

by (7).

Relations such as (12) and (16) hold for the process $w_{n}(t)$. According to [8], there exist a subsequence $n^{\prime} \rightarrow \infty$, a probability space $(\tilde{\Omega}, \tilde{\mathcal{F}}, \tilde{\mathrm{P}})$, and random vectors

$\tilde{\eta}_{n^{\prime}}(t)=\left(\tilde{\zeta}_{n^{\prime}}(t), \tilde{\alpha}_{n^{\prime}}(t), \tilde{\gamma}_{n^{\prime}}(t), \tilde{\beta}_{n^{\prime}}(t), \tilde{w}_{n^{\prime}}(t)\right), \quad \tilde{\eta}_{0}(t)=\left(\tilde{\zeta}_{0}(t), \tilde{\alpha}_{0}(t), \tilde{\gamma}_{0}(t), \tilde{\beta}_{0}(t), \tilde{w}_{0}(t)\right)$

defined on this probability space such that all finite-dimensional distributions of the process $\tilde{\eta}_{n^{\prime}}(t)$ coincide with those of the process

$$
\eta_{n^{\prime}}(t)=\left(\zeta_{n^{\prime}}(t), \alpha_{n^{\prime}}(t), \gamma_{n^{\prime}}(t), \beta_{n^{\prime}}(t), w_{n^{\prime}}(t)\right)
$$

and, for all $t \geq 0$, the components of the process $\tilde{\eta}_{n^{\prime}}(t)$ converge in probability as $n^{\prime} \rightarrow \infty$ to the corresponding components of the process $\tilde{\eta}_{0}(t)$. One can show (see [3]) that the relations between components of the process $\eta_{n^{\prime}}(t)$ hold for the components of the process $\tilde{\eta}_{n^{\prime}}(t)$, as well. For the sake of simplicity, we assume in what follows that $n^{\prime}=n$ and that the components of the process $\eta_{n}(t)$ converge in probability as $n \rightarrow \infty$ to the corresponding components of the process $\eta_{0}(t)=\left(\zeta_{0}(t), \alpha_{0}(t), \gamma_{0}(t), \beta_{0}(t), w_{0}(t)\right)$. Since the process $\beta_{n}(t)$ is expressed in a form similar to (9),

$$
\begin{aligned}
\mathrm{P}\left\{\sup _{0 \leq t \leq 1}\left|\beta_{n}(t)\right|>\varepsilon\right\} & \leq \mathrm{P}\left\{\sup _{0 \leq k \leq k_{n}-1}\left[2\left|\beta_{n k}\right|+\left|\beta_{n k+1}\right|\right]>\varepsilon\right\} \\
& \leq \mathrm{P}\left\{\sup _{0 \leq k \leq k_{n}}\left|\beta_{n k}\right|>\frac{\varepsilon}{3}\right\} \leq\left(\frac{3}{\varepsilon}\right)^{2} \mathrm{E} \sup _{0 \leq k \leq k_{n}}\left|\beta_{n k}\right|^{2} .
\end{aligned}
$$


According to (7),

$$
\sup _{0 \leq t \leq 1}\left|\beta_{n}(t)\right| \stackrel{\mathrm{P}}{\rightarrow} 0
$$

as $n \rightarrow \infty$. Thus the process $\beta_{n}(t)$ weakly converges to zero as $n \rightarrow \infty$ (see [1]).

We need the following auxiliary results.

Lemma 3.1. Let conditions $\left(I_{1}\right)-\left(I_{6}\right)$ hold. Then

$$
\lim _{C \rightarrow \infty} \varlimsup_{n \rightarrow \infty} \mathrm{P}\left\{\sup _{0 \leq t \leq 1}\left|\zeta_{n}(t)\right|>C\right\}=0 .
$$

Proof. Similarly to (11) we prove that

$$
\mathrm{P}\left\{\sup _{0 \leq t \leq 1}\left|\zeta_{n}(t)\right|>C\right\} \leq \mathrm{P}\left\{3 \sup _{0 \leq k \leq k_{n}}\left|\zeta_{n k}\right|>C\right\} \leq\left(\frac{3}{C}\right)^{2} \mathrm{E} \sup _{0 \leq k \leq k_{n}}\left|\zeta_{n k}\right|^{2} \leq\left(\frac{3}{C}\right)^{2} \hat{C},
$$

which completes the proof of Lemma 3.1 .

Lemma 3.2. Let $|g(x)| \leq C$ and let condition $\left(I_{7}\right)$ hold. Then

$$
\varlimsup_{n \rightarrow \infty} \mathrm{E}\left|\sum_{t^{\prime}<t_{n k}<t^{\prime \prime}} g\left(\xi_{n k}\right) \Delta \omega_{n k}\right|^{4} \leq 8 C^{4}\left|t^{\prime \prime}-t^{\prime}\right|^{2}
$$

for all $0 \leq t^{\prime}<t^{\prime \prime} \leq 1$.

Proof. Put $g_{n k}=g\left(\xi_{n k}\right) \Delta \omega_{n k}$ and let $t_{n i}=\min _{k}\left\{t_{n k} \geq t^{\prime}\right\}$ and $t_{n j}=\max _{k}\left\{t_{n k} \leq t^{\prime \prime}\right\}$. We have

$$
\begin{aligned}
\left|\sum_{k=i}^{j} g_{n k}\right|^{4}= & 4 \sum_{l=i}^{j-1}\left(\sum_{k=i}^{l} g_{n k}\right)^{3} g_{n l+1}+6 \sum_{l=i}^{j-1}\left(\sum_{k=i}^{l} g_{n k}\right)^{2}\left(g_{n l+1}\right)^{2} \\
& +4 \sum_{l=i}^{j-1}\left(\sum_{k=i}^{l} g_{n k}\right)\left(g_{n l+1}\right)^{3}+\sum_{l=i}^{j}\left(g_{n l}\right)^{4} \\
= & B_{1}+B_{2}+B_{3}+B_{4}
\end{aligned}
$$

and $\mathrm{E} B_{1}=0$. Since

$$
\begin{aligned}
\mathrm{E} B_{2} & =6 \sum_{l=i}^{j-1} \mathrm{E}\left(\sum_{k=i}^{l} g_{n k}\right)^{2} g^{2}\left(\xi_{n l+1}\right) \Delta t_{n l+1} \leq 6 C^{2} \sum_{l=i}^{j-1}\left(\sum_{k=i}^{l} \mathrm{E} g^{2}\left(\xi_{n k}\right) \Delta t_{n k}\right) \Delta t_{n l+1} \\
& \leq 6 C^{4}\left|t^{\prime \prime}-t^{\prime}\right|^{2}, \\
& \mathrm{E} B_{4} \leq C^{4} \sum_{l=i}^{j} \mathrm{E}\left(\Delta \omega_{n l}\right)^{4} \leq 8 C^{4}\left[\sum_{l=0}^{k_{n}-1} \mathrm{E}\left|\frac{\Delta \xi_{n l}}{\sigma_{n}\left(\xi_{n l}\right)}\right|^{4}+\lambda_{n}^{3} \sup _{x}\left|\frac{a_{n}(x)}{\sigma_{n}(x)}\right|^{4}\right] \rightarrow 0
\end{aligned}
$$

as $n \rightarrow \infty$, and

$$
\begin{aligned}
\left|\mathrm{E} B_{3}\right| & =4\left|\mathrm{E} \sum_{l=i}^{j-1}\left(\sum_{k=i}^{l} g_{n k}\right) g_{n l+1} g_{n l+1}^{2}\right| \leq 2 \mathrm{E} \sum_{l=i}^{j-1}\left[\left(\sum_{k=1}^{l} g_{n k}\right)^{2}+g_{n l+1}^{2}\right] g_{n l+1}^{2} \\
& =2 \mathrm{E} \sum_{l=i}^{j-1}\left(\sum_{k=1}^{l} g_{n k}\right)^{2} g_{n l+1}^{2}+2 \mathrm{E} \sum_{l=i}^{j-1} g_{n l+1}^{4} \leq \frac{1}{3} \mathrm{E} B_{2}+2 \mathrm{E} B_{4} \\
& \leq 2 C^{4}\left|t^{\prime \prime}-t^{\prime}\right|^{2}+o(1)
\end{aligned}
$$


where $o(1)$ is a variable approaching zero as $n \rightarrow \infty$, we obtain

$$
\mathrm{E}\left|\sum_{k=i}^{j} g_{n k}\right|^{4} \leq 8 C^{4}\left|t^{\prime \prime}-t^{\prime}\right|^{2}+o(1) .
$$

The latter inequality completes the proof of Lemma 3.2

Lemma 3.3. Let condition $\left(I_{7}\right)$ hold. Then the limit $w_{0}(t)$ is a Wiener process.

Proof. It is clear that the process $w_{n}(t)$ is a square integrable martingale for all $n>1$ with respect to the $\sigma$-algebra $\sigma\left\{w_{n}(s), s \leq t\right\}$ and that its characteristic is

$$
\left\langle w_{n}(t)\right\rangle=t_{n k+1} \quad \text { for } t \in\left[t_{n k}, t_{n k+1}\right] .
$$

Thus $w_{n}(t) \stackrel{\mathrm{P}}{\rightarrow} w_{0}(t)$ and $\left\langle w_{n}(t)\right\rangle \stackrel{\mathrm{P}}{\rightarrow} t$ as $n \rightarrow \infty$. According to Lemma 3.2 with the function $g(x)=1$,

$$
\sup _{0 \leq t \leq 1} \mathrm{E} w_{n}^{4}(t) \leq C
$$

Thus $w_{0}(t)$ is a martingale with respect to the minimal $\sigma$-algebra $\sigma\left\{w_{0}(s), s \leq t\right\}$ and its characteristic is $\left\langle w_{0}(t)\right\rangle=t$ (see [7]). Moreover,

$$
\varlimsup_{n \rightarrow \infty} \mathrm{E}\left|w_{n}\left(t^{\prime \prime}\right)-w_{n}\left(t^{\prime}\right)\right|^{4} \leq C\left|t^{\prime \prime}-t^{\prime}\right|^{2}
$$

for all $0 \leq t^{\prime}<t^{\prime \prime} \leq 1$ by Lemma 3.2. Now Fatou's lemma implies

$$
\mathrm{E}\left|w_{0}\left(t^{\prime \prime}\right)-w_{0}\left(t^{\prime}\right)\right|^{4} \leq C\left|t^{\prime \prime}-t^{\prime}\right|^{2} .
$$

Thus the martingale $w_{0}(t)$ is continuous with probability one (see [1]). This implies that $w_{0}(t)$ is a Wiener process 2 .

Lemma 3.4. Let conditions $\left(I_{2}\right)-\left(I_{7}\right)$ hold. Assume that

$$
\left|L G_{n}(x)\right|^{2}+\left|G_{n}^{\prime}(x) \sigma_{n}(x)\right|^{2} \leq K, \quad G_{n}^{\prime}(x) \sigma_{n}(x) \geq \delta>0 .
$$

Then

$$
\lim _{\varepsilon \rightarrow 0} \varlimsup_{n \rightarrow \infty} \sum_{k=0}^{k_{n}-1} \mathrm{P}\left\{\zeta_{n k} \in A^{(\varepsilon)}\right\} \Delta t_{n k}=0
$$

for an arbitrary collection of points $x_{j}, j=1, \ldots, m$.

Proof. Consider the function $Q(x)=2 \int_{0}^{x} \int_{0}^{u} q_{\varepsilon+\varepsilon_{1}}(v) d v d u$, where $\varepsilon>0, \varepsilon_{1}>0$, and $q_{\varepsilon+\varepsilon_{1}}(x)$ is a nonnegative continuously differentiable function such that $q_{\varepsilon+\varepsilon_{1}}(x)=1$ for $x \in A^{(\varepsilon)}$ and $q_{\varepsilon+\varepsilon_{1}}(x)=0$ for $x \in \overline{A^{\left(\varepsilon+\varepsilon_{1}\right)}}$. We also require that $\left|q_{\varepsilon+\varepsilon_{1}}(x)\right| \leq 1$. Then the Taylor formula yields

$$
\begin{aligned}
\sum_{k=0}^{k_{n}-1} q_{\varepsilon} & \varepsilon_{1}\left(\hat{\zeta}_{n k}\right)\left[G_{n}^{\prime}\left(\xi_{n k}\right) \sigma_{n}\left(\xi_{n k}\right)\right]^{2} \Delta t_{n k} \\
= & {\left[Q\left(\hat{\zeta}_{n k_{n}}\right)-Q\left(\hat{\zeta}_{n 0}\right)\right]-\sum_{k=0}^{k_{n}-1} Q^{\prime}\left(\hat{\zeta}_{n k}\right) \Delta \alpha_{n k}-\sum_{k=0}^{k_{n}-1} Q^{\prime}\left(\hat{\zeta}_{n k}\right) \Delta \gamma_{n k} } \\
& -\frac{1}{2} \sum_{k=0}^{k_{n}-1} Q^{\prime \prime}\left(\hat{\zeta}_{n k}\right)\left(\Delta \alpha_{n k}\right)^{2}-\sum_{k=0}^{k_{n}-1} Q^{\prime \prime}\left(\hat{\zeta}_{n k}\right) \Delta \alpha_{n k} \Delta \gamma_{n k} \\
& -\frac{1}{2} \sum_{k=0}^{k_{n}-1} Q^{\prime \prime}\left(\hat{\zeta}_{n k}\right)\left[\left(\Delta \gamma_{n k}\right)^{2}-\left[G_{n}^{\prime}\left(\xi_{n k}\right) \sigma_{n}\left(\xi_{n k}\right)\right]^{2} \Delta t_{n k}\right] \\
& -\frac{1}{2} \sum_{k=0}^{k_{n}-1}\left[Q^{\prime \prime}\left(\hat{\zeta}_{n k}+\theta \Delta \hat{\zeta}_{n k}\right)-Q^{\prime \prime}\left(\hat{\zeta}_{n k}\right)\right]\left(\Delta \hat{\zeta}_{n k}\right)^{2}=\sum_{l=1}^{7} J_{l}
\end{aligned}
$$


where $\hat{\zeta}_{n k}=\zeta_{n 0}+\alpha_{n k}+\gamma_{n k}, \Delta \hat{\zeta}_{n k}=\hat{\zeta}_{n k+1}-\hat{\zeta}_{n k}, \Delta \alpha_{n k}=\alpha_{n k+1}-\alpha_{n k}, \Delta \gamma_{n k}=$ $\gamma_{n k+1}-\gamma_{n k}$, and $0<\theta<1$. Here $J_{l}, 1 \leq l \leq 7$, denote the corresponding terms of the middle part of relation (21). It is clear that

$$
|Q(x)-Q(y)| \leq 4 m\left(\varepsilon+\varepsilon_{1}\right)|x-y|, \quad\left|Q^{\prime}(x)\right| \leq 4 m\left(\varepsilon+\varepsilon_{1}\right),
$$

and that $Q^{\prime \prime}(x)$ is a uniformly continuous function in the domain $|x| \leq N$. Thus

$$
\begin{aligned}
\left|\mathrm{E} J_{1}\right| & \leq 4 m\left(\varepsilon+\varepsilon_{1}\right) \mathrm{E}\left|\hat{\zeta}_{n k_{n}}-\hat{\zeta}_{n 0}\right|=4 m\left(\varepsilon+\varepsilon_{1}\right) \mathrm{E}\left|\alpha_{n k_{n}}+\gamma_{n k_{n}}\right| \\
& \leq 4 m\left(\varepsilon+\varepsilon_{1}\right)\left[\mathrm{E}\left|\zeta_{n 0}\right|+\mathrm{E}\left|\zeta_{n k_{n}}\right|+\mathrm{E}\left|\beta_{n k_{n}}\right|\right] \leq C\left(\varepsilon+\varepsilon_{1}\right), \\
\left|\mathrm{E} J_{2}\right| & \leq 4 m\left(\varepsilon+\varepsilon_{1}\right) \sum_{k=0}^{k_{n}-1} \mathrm{E}\left|L G_{n}\left(\xi_{n k}\right)\right| \Delta t_{n k} \leq 4 \sqrt{K} m\left(\varepsilon+\varepsilon_{1}\right) .
\end{aligned}
$$

Moreover $\mathrm{E} J_{3}=0, \mathrm{E} J_{5}=0, \mathrm{E} J_{6}=0$, and

$$
\left|\mathrm{E} J_{4}\right| \leq \frac{1}{2} \sum_{k=0}^{k_{n}-1} \mathrm{E}\left|L G_{n}\left(\xi_{n k}\right)\right|^{2}\left(\Delta t_{n k}\right)^{2} \leq \lambda_{n} \frac{1}{2} K .
$$

We use the uniform continuity of $Q^{\prime \prime}(x)$ to estimate $\left|\mathrm{E} J_{7}\right|$. Namely, for an arbitrary $\varepsilon_{0}>0$, there exists $\delta_{0}>0$ such that

$$
\left|Q^{\prime \prime}\left(\hat{\zeta}_{n k}+\theta \Delta \hat{\zeta}_{n k}\right)-Q^{\prime \prime}\left(\hat{\zeta}_{n k}\right)\right|<\varepsilon_{0}
$$

if $\left|\Delta \hat{\zeta}_{n k}\right|<\delta_{0}$. Hence

$$
\begin{aligned}
\left|\mathrm{E} J_{7}\right| \leq & \frac{1}{2} \varepsilon_{0} \sum_{k=0}^{k_{n}-1} \mathrm{E}\left|\Delta \hat{\zeta}_{n k}\right|^{2}+\sum_{k=0}^{k_{n}-1} \mathrm{E} \chi_{|x| \geq \delta_{0}}\left(\Delta \hat{\zeta}_{n k}\right)\left|\Delta \hat{\zeta}_{n k}\right|^{2} \\
\leq & \varepsilon_{0} \sum_{k=0}^{k_{n}-1} \mathrm{E}\left\{\left[L G_{n}\left(\xi_{n k}\right)\right]^{2}\left(\Delta t_{n k}\right)^{2}+\left[G_{n}^{\prime}\left(\xi_{n k}\right) \sigma_{n}\left(\xi_{n k}\right)\right]^{2}\left(\Delta \omega_{n k}\right)^{2}\right\} \\
& +\sum_{k=0}^{k_{n}-1}\left(\mathrm{P}\left\{\left|\Delta \hat{\zeta}_{n k}\right| \geq \delta_{0}\right\}\right)^{1 / 2}\left(\mathrm{E}\left|\Delta \hat{\zeta}_{n k}\right|^{4}\right)^{1 / 2} \\
\leq & \varepsilon_{0}\left[\lambda_{n} \cdot K+K\right]+\sum_{k=0}^{k_{n}-1}\left(\frac{1}{\delta_{0}^{4}} \mathrm{E}\left|\Delta \hat{\zeta}_{n k}\right|^{4}\right)^{1 / 2}\left(\mathrm{E}\left|\Delta \hat{\zeta}_{n k}\right|^{4}\right)^{1 / 2} \\
\leq & \varepsilon_{0} K\left(\lambda_{n}+1\right)+\frac{1}{\delta_{0}^{2}} \sum_{k=0}^{k_{n}-1} \mathrm{E}\left|\Delta \hat{\zeta}_{n k}\right|^{4} \leq 2 \varepsilon_{0} K+\frac{8}{\delta_{0}^{2}} \sum_{k=0}^{k_{n}-1}\left(\mathrm{E}\left|\Delta \alpha_{n k}\right|^{4}+\mathrm{E}\left|\Delta \gamma_{n k}\right|^{4}\right) \\
\leq & 2 \varepsilon_{0} K+\frac{8}{\delta_{0}^{2}} K^{2} \lambda_{n}^{3}+\frac{8}{\delta_{0}^{2}} K^{2} \sum_{k=0}^{k_{n}-1} \mathrm{E}\left|\Delta \omega_{n k}\right|^{4} \\
\leq & 2 \varepsilon_{0} K+\frac{8}{\delta_{0}^{2}} K^{2} \lambda_{n}^{3}+\frac{64}{\delta_{0}^{2}} K^{2} \sum_{k=0}^{k_{n}-1}\left(\mathrm{E}\left|\frac{a_{n}\left(\xi_{n k}\right)}{\sigma_{n}\left(\xi_{n k}\right)}\right|^{4}\left(\Delta t_{n k}\right)^{4}+\mathrm{E}\left|\frac{\Delta \xi_{n k}}{\sigma_{n}\left(\xi_{n k}\right)}\right|^{4}\right) \\
\leq & 2 \varepsilon_{0} K+\frac{8}{\delta_{0}^{2}} K^{2} \lambda_{n}^{3}+\frac{64}{\delta_{0}^{2}} K^{2} \lambda_{n}^{3} \sup \left|\frac{a_{n}(x)}{\sigma_{n}(x)}\right|^{4}+\frac{64}{\delta_{0}^{2}} K^{2} \sum_{k=0}^{k_{n}-1} \mathrm{E}\left|\frac{\Delta \xi_{n k}}{\sigma_{n}\left(\xi_{n k}\right)}\right|^{4} \\
= & 2 \varepsilon_{0} K+o(1) .
\end{aligned}
$$

Therefore $\varlimsup_{n \rightarrow \infty}\left|\mathrm{E} J_{7}\right| \leq 2 \varepsilon_{0} K$. 
Since $\varepsilon_{0}>0$ is an arbitrary number, the latter inequality means that the limit $\lim _{n \rightarrow \infty} \mathrm{E} J_{7}$ exists and is zero. In view of representation (21),

$$
\varlimsup_{n \rightarrow \infty} \mathrm{E} \sum_{k=0}^{k_{n}-1} q_{\varepsilon+\varepsilon_{1}}\left(\hat{\zeta}_{n k}\right)\left[G_{n}^{\prime}\left(\xi_{n k}\right) \sigma_{n}\left(\xi_{n k}\right)\right]^{2} \Delta t_{n k} \leq C\left(\varepsilon+\varepsilon_{1}\right) .
$$

Finally, we take into account the inequalities

$$
\begin{aligned}
& \sum_{k=0}^{k_{n}-1} \mathrm{P}\left\{\zeta_{n k} \in A^{(\varepsilon)}\right\} \Delta t_{n k} \leq \sum_{k=0}^{k_{n}-1} \mathrm{E} q_{\varepsilon+\varepsilon_{1}}\left(\zeta_{n k}\right) \Delta t_{n k} \\
& \quad \leq \sum_{k=0}^{k_{n}-1} \mathrm{E}\left|q_{\varepsilon+\varepsilon_{1}}\left(\zeta_{n k}\right)-q_{\varepsilon+\varepsilon_{1}}\left(\hat{\zeta}_{n k}\right)\right| \Delta t_{n k}+\sum_{k=0}^{k_{n}-1} \mathrm{E} q_{\varepsilon+\varepsilon_{1}}\left(\hat{\zeta}_{n k}\right) \Delta t_{n k} \\
& \quad \leq \sup _{x}\left|q_{\varepsilon+\varepsilon_{1}}^{\prime}(x)\right| \sup _{x}\left|\beta_{n k}\right|+\frac{1}{\delta^{2}} \mathrm{E} \sum_{k=0}^{k_{n}-1} q_{\varepsilon+\varepsilon_{1}}\left(\hat{\zeta}_{n k}\right)\left[G_{n}^{\prime}\left(\xi_{n k}\right) \sigma_{n}\left(\xi_{n k}\right)\right]^{2} \Delta t_{n k}
\end{aligned}
$$

and bound (7) to complete the proof of Lemma 3.4

Lemma 3.5. Let the assumptions of Lemma 3.4 be satisfied. Then

$$
\lim _{\varepsilon \rightarrow 0} \varlimsup_{n \rightarrow \infty} \int_{0}^{1} \mathrm{P}\left\{\zeta_{n}(s) \in A^{(\varepsilon)}\right\} d s=0 .
$$

Proof. Consider a number $\varepsilon$ such that $\left\{x:\left|x-x_{j}\right|<2 \varepsilon\right\} \cap\left\{x:\left|x-x_{k}\right|<2 \varepsilon\right\}=\varnothing$ for $j \neq k$. Then

$$
\begin{aligned}
\int_{0}^{1} \mathrm{P}\left\{\zeta_{n}(s) \in A^{(\varepsilon)}\right\} d s= & \sum_{i=0}^{k_{n}-1} \int_{t_{n i}}^{t_{n i+1}} \mathrm{P}\left\{\zeta_{n}(s) \in A^{(\varepsilon)}\right\} d s \\
\leq & \sum_{i=0}^{k_{n}-1} \int_{t_{n i}}^{t_{n i+1}} \mathrm{E} \chi_{A^{(\varepsilon)}}\left(\zeta_{n}(s)\right) \chi_{A^{(2 \varepsilon)}}\left(\zeta_{n}\left(t_{n i}\right)\right) d s \\
& +\sum_{i=0}^{k_{n}-1} \int_{t_{n i}}^{t_{n i+1}} \mathrm{E} \chi_{A^{(\varepsilon)}}\left(\zeta_{n}(s)\right) \chi_{\overline{A^{(2 \varepsilon)}}}\left(\zeta_{n}\left(t_{n i}\right)\right) d s \\
\leq & \sum_{i=0}^{k_{n}-1} \mathrm{P}\left\{\zeta_{n i} \in A^{(2 \varepsilon)}\right\} \Delta t_{n i}+\sup _{\left|t^{\prime \prime}-t^{\prime}\right| \leq h} \mathrm{P}\left\{\left|\zeta_{n}\left(t^{\prime \prime}\right)-\zeta_{n}\left(t^{\prime}\right)\right|>\varepsilon\right\}
\end{aligned}
$$

for $\lambda_{n}<h$. Passing to the limit in the latter relation as $n \rightarrow \infty$, then as $h \rightarrow 0$, and finally as $\varepsilon \rightarrow 0$, we complete the proof of Lemma 3.5 by Lemma 3.4 and convergence (16).

Theorem 3.1. Let conditions $\left(I_{1}\right)-\left(I_{7}\right)$ hold. If there exist continuous functions $a_{0}(y)$ and $\sigma_{0}(y)$ such that

1) $\lim _{n \rightarrow \infty} \sum_{k=0}^{k_{n}-1} \mathrm{E}\left|L G_{n}\left(\xi_{n k}\right)-a_{0}\left(G_{n}\left(\xi_{n k}\right)\right)\right| \Delta t_{n k}=0$,

2) $\lim _{n \rightarrow \infty} \sum_{k=0}^{k_{n}-1} \mathrm{E}\left[G_{n}^{\prime}\left(\xi_{n k}\right) \sigma_{n}\left(\xi_{n k}\right)-\sigma_{0}\left(G_{n}\left(\xi_{n k}\right)\right)\right]^{2} \Delta t_{n k}=0$,

3) the stochastic equation

$$
\zeta_{0}(t)=\zeta_{0}+\int_{0}^{t} a_{0}\left(\zeta_{0}(s)\right) d s+\int_{0}^{t} \sigma_{0}\left(\zeta_{0}(s)\right) d w_{0}(s)
$$

has a unique weak solution,

then the random polygonal line $\zeta_{n}(t)$ weakly converges as $n \rightarrow \infty$ to the solution $\zeta_{0}(t)$ of equation (22). 
Proof. First we show that

$$
\sup _{0 \leq t \leq 1}\left|\alpha_{n}(t)-\int_{0}^{t} a_{0}\left(\zeta_{n}(s)\right) d s\right| \stackrel{\mathrm{P}}{\rightarrow} 0
$$

and

$$
\sup _{0 \leq t \leq 1}\left|\gamma_{n}(t)-\int_{0}^{t} \sigma_{0}\left(\zeta_{n}(s)\right) d w_{n}(s)\right| \stackrel{\mathrm{P}}{\rightarrow} 0
$$

as $n \rightarrow \infty$. To prove (23) we use the inequality

$$
\sup _{0 \leq t \leq 1}\left|\alpha_{n}(t)-\int_{0}^{t} a_{0}\left(\zeta_{n}(s)\right) d s\right| \leq S_{n}^{(1)}+S_{n}^{(2)},
$$

where

$$
\begin{gathered}
S_{n}^{(1)}=\sum_{i=0}^{k_{n}-1}\left|L G_{n}\left(\xi_{n i}\right)-a_{0}\left(\zeta_{n i}\right)\right| \Delta t_{n i}, \\
S_{n}^{(2)}=\sum_{i=0}^{k_{n}-1} \int_{t_{n i}}^{t_{n i+1}}\left|a_{0}\left(\zeta_{n}(s)\right)-a_{0}\left(\zeta_{n}\left(t_{n i}\right)\right)\right| d s .
\end{gathered}
$$

By the assumptions of the theorem, $S_{n}^{(1)} \stackrel{\mathrm{P}}{\rightarrow} 0$ as $n \rightarrow \infty$. Since the function $a_{0}(x)$ is continuous, it is uniformly continuous in the domain $|x| \leq N$. Thus, for an arbitrary $\varepsilon_{0}>0$, there exists $\delta_{0}>0$ such that $\left|a_{0}\left(x_{1}\right)-a_{0}\left(x_{2}\right)\right|<\varepsilon_{0}$ if $\left|x_{1}-x_{2}\right| \leq \delta_{0}$. Therefore, for an arbitrary $\varepsilon>0$,

$$
\begin{aligned}
\mathrm{P}\left\{S_{n}^{(2)}>\varepsilon\right\} & \leq P_{N}+\mathrm{P}\left\{\sum_{i=0}^{k_{n}-1} \int_{t_{n i}}^{t_{n i+1}}\left|a_{0}\left(\zeta_{n}(s)\right)-a_{0}\left(\zeta_{n}\left(t_{n i}\right)\right)\right| \chi_{|x| \leq N}\left(\zeta_{n}(s)\right) d s>\frac{\varepsilon}{2}\right\} \\
& \leq P_{N}+\frac{2}{\varepsilon} \mathrm{E} \sum_{i=0}^{k_{n}-1} \int_{t_{n i}}^{t_{n i+1}}\left|a_{0}\left(\zeta_{n}(s)\right)-a_{0}\left(\zeta_{n}\left(t_{n i}\right)\right)\right| \chi_{|x| \leq N}\left(\zeta_{n}(s)\right) d s \\
& \leq P_{N}+\frac{2}{\varepsilon} \cdot \varepsilon_{0}+\frac{2}{\varepsilon} C_{N} \sum_{i=0}^{k_{n}-1} \int_{t_{n i}}^{t_{n i+1}} \mathrm{P}\left\{\left|\zeta_{n}(s)-\zeta_{n}\left(t_{n i}\right)\right|>\delta_{0}\right\} d s
\end{aligned}
$$

Considering (16) we obtain

$$
\varlimsup_{n \rightarrow \infty} \mathrm{P}\left\{S_{n}^{(2)}>\varepsilon\right\} \leq \varlimsup_{n \rightarrow \infty} P_{N}+\frac{2}{\varepsilon} \cdot \varepsilon_{0}
$$

Since $\varepsilon_{0}$ is arbitrary,

$$
\varlimsup_{n \rightarrow \infty} \mathrm{P}\left\{S_{n}^{(2)}>\varepsilon\right\} \leq \varlimsup_{n \rightarrow \infty} P_{N}
$$

for all $N>0$. By Lemma 3.1. $P_{N} \rightarrow 0$ as $N \rightarrow \infty$, whence we derive that $S_{n}^{(2)} \stackrel{\mathrm{P}}{\rightarrow} 0$ as $n \rightarrow \infty$. This result proves convergence (23).

To prove convergence (24) note that

$$
\sup _{0 \leq t \leq 1}\left|\gamma_{n}(t)-\int_{0}^{t} \sigma_{0}\left(\zeta_{n}(s)\right) d w_{n}(s)\right| \leq \sup _{k}\left|\tilde{S}_{n}^{(1)}\right|+\sup _{k} \sup _{t \in\left[t_{n k}, t_{n k+1}\right)}\left|\tilde{S}_{n}^{(2)}\right|,
$$

where

$$
\begin{gathered}
\tilde{S}_{n}^{(1)}=\sum_{i=0}^{k-1}\left[G_{n}^{\prime}\left(\xi_{n i}\right) \sigma_{n}\left(\xi_{n i}\right)-\sigma_{0}\left(\zeta_{n i}\right)\right] \Delta \omega_{n i} \\
\tilde{S}_{n}^{(2)}=\frac{t-t_{n k}}{\Delta t_{n k}} G_{n}^{\prime}\left(\xi_{n k}\right) \sigma_{n}\left(\xi_{n k}\right) \Delta \omega_{n k}-\sigma_{0}\left(\zeta_{n k}\right) \Delta \omega_{n k} .
\end{gathered}
$$


Since $\tilde{S}_{n}^{(1)}$ is a square integrable martingale in $k$, we have

$$
\begin{aligned}
\sup _{k}\left|\tilde{S}_{n}^{(1)}\right| & \leq 4 \mathrm{E}\left(\sum_{i=0}^{k_{n}-1}\left[G_{n}^{\prime}\left(\xi_{n i}\right) \sigma_{n}\left(\xi_{n i}\right)-\sigma_{0}\left(\zeta_{n i}\right)\right] \Delta \omega_{n i}\right)^{2} \\
& =4 \mathrm{E} \sum_{i=0}^{k_{n}-1} \mathrm{E}\left|G_{n}^{\prime}\left(\xi_{n i}\right) \sigma_{n}\left(\xi_{n i}\right)-\sigma_{0}\left(\zeta_{n i}\right)\right|^{2} \Delta t_{n i}
\end{aligned}
$$

By the assumptions of the theorem, $\operatorname{Esup}_{k}\left|\tilde{S}_{n}^{(1)}\right| \rightarrow 0$ as $n \rightarrow \infty$, whence $\sup _{k}\left|\tilde{S}_{n}^{(1)}\right| \stackrel{\mathrm{P}}{\rightarrow} 0$ as $n \rightarrow \infty$. The bounds

$$
\begin{aligned}
& P\left\{\sup _{k} \sup _{t \in\left[t_{n k}, t_{n k+1}\right)}\left|\tilde{S}_{n}^{(2)}\right|>\varepsilon\right\} \\
& \leq \mathrm{P}\left\{\sup _{k}\left|\zeta_{n k}\right|>N\right\} \\
& +\mathrm{P}\left\{\sup _{k} \sup _{t \in\left[t_{n k}, t_{n k+1}\right)}\left|\left[\frac{t-t_{n k}}{\Delta t_{n k}} G_{n}^{\prime}\left(\xi_{n k}\right) \sigma_{n}\left(\xi_{n k}\right)-\sigma_{0}\left(\zeta_{n k}\right)\right] \chi_{|x| \leq N}\left(\zeta_{n k}\right) \Delta \omega_{n k}\right|>\frac{\varepsilon}{2}\right\} \\
\leq & P_{N}+\left(\frac{2}{\varepsilon}\right)^{4} 8 \mathrm{E} \sup _{k}\left(\left|G_{n}^{\prime}\left(\xi_{n k}\right) \sigma_{n}\left(\xi_{n k}\right)\right|^{4}+\left|\sigma_{0}\left(\zeta_{n k}\right)\right|^{4}\right) \chi_{|x| \leq N}\left(\zeta_{n k}\right)\left|\Delta \omega_{n k}\right|^{4} \\
\leq & P_{N}+\left(\frac{2}{\varepsilon}\right)^{4} C_{N} \mathrm{E} \sum_{i=0}^{k_{n}-1}\left|\Delta \omega_{n i}\right|^{4} \\
\leq & P_{N}+\left(\frac{2}{\varepsilon}\right)^{4} C_{N}\left[8 \sum_{i=0}^{k_{n}-1} \mathrm{E}\left|\frac{\Delta \xi_{n i}}{\sigma_{n}\left(\xi_{n i}\right)}\right|^{4}+8 \lambda_{n}^{3} \sup _{x}\left|\frac{a_{n}(x)}{\sigma_{n}(x)}\right|^{4}\right]
\end{aligned}
$$

imply

$$
\varlimsup_{n \rightarrow \infty} \mathrm{P}\left\{\sup _{k}\left|\tilde{S}_{n}^{(2)}\right|>\varepsilon\right\} \leq P_{N}
$$

Passing to the limit as $N \rightarrow \infty$ we obtain

$$
\sup _{k}\left|\tilde{S}_{n}^{(2)}\right| \stackrel{\text { P }}{\rightarrow} 0 \quad \text { as } n \rightarrow \infty
$$

This proves convergence (24). It remains to show that

$$
\sup _{0 \leq t \leq 1}\left|\int_{0}^{t} a_{0}\left(\zeta_{n}(s)\right) d s-\int_{0}^{t} a_{0}\left(\zeta_{0}(s)\right) d s\right| \stackrel{\mathrm{P}}{\rightarrow} 0
$$

and

$$
\sup _{0 \leq t \leq 1}\left|\int_{0}^{t} \sigma_{0}\left(\zeta_{n}(s)\right) d w_{n}(s)-\int_{0}^{t} \sigma_{0}\left(\zeta_{0}(s)\right) d w_{0}(s)\right| \stackrel{\mathrm{P}}{\rightarrow} 0
$$

as $n \rightarrow \infty$ and to use equality (10) to complete the proof of Theorem 3.1 ,

Consider the continuous function $q_{N}(x)$ that is equal to 1 for $|x| \leq N$, vanishes for $|x| \geq N+1$, and satisfies $q_{N}(x)=-|x|+1+N$ for $N<|x|<N+1$. 
Then, for an arbitrary $\varepsilon>0$,

$$
\begin{aligned}
& \mathrm{P}\left\{\sup _{0 \leq t \leq 1}\left|\int_{0}^{t}\left[a_{0}\left(\zeta_{n}(s)\right)-a_{0}\left(\zeta_{0}(s)\right)\right] d s\right|>\varepsilon\right\} \\
& \leq \mathrm{P}\left\{\sup _{0 \leq t \leq 1}\left|\int_{0}^{t} a_{0}\left(\zeta_{n}(s)\right) q_{N}\left(\zeta_{n}(s)\right) d s-\int_{0}^{t} a_{0}\left(\zeta_{0}(s)\right) q_{N}\left(\zeta_{0}(s)\right) d s\right|>\frac{\varepsilon}{3}\right\} \\
& \quad+\mathrm{P}\left\{\sup _{0 \leq t \leq 1}\left|\int_{0}^{t} a_{0}\left(\zeta_{n}(s)\right)\left[1-q_{N}\left(\zeta_{n}(s)\right)\right] d s\right|>\frac{\varepsilon}{3}\right\} \\
& \quad+\mathrm{P}\left\{\sup _{0 \leq t \leq 1}\left|\int_{0}^{t} a_{0}\left(\zeta_{0}(s)\right)\left[1-q_{N}\left(\zeta_{0}(s)\right)\right] d s\right|>\frac{\varepsilon}{3}\right\} \\
& \leq o(1)+P_{N}+\mathrm{P}\left\{\sup _{0 \leq t \leq 1}\left|\zeta_{0}(t)\right|>N\right\}=o(1)+P_{N}+\varlimsup_{n \rightarrow \infty} P_{N},
\end{aligned}
$$

where $o(1)$ is a variable approaching zero as $n \rightarrow \infty$ for all $\varepsilon>0$. Since $a_{0}(x) q_{N}(x)$ is a bounded continuous function and

$$
\zeta_{n}(s) \stackrel{\mathrm{P}}{\rightarrow} \zeta_{0}(s) \text { as } n \rightarrow \infty,
$$

we have

$$
\int_{0}^{t} a_{0}\left(\zeta_{n}(s)\right) q_{N}\left(\zeta_{n}(s)\right) d s \stackrel{\mathrm{P}}{\rightarrow} \int_{0}^{t} a_{0}\left(\zeta_{0}(s)\right) q_{N}\left(\zeta_{0}(s)\right) d s
$$

for all $N>0$. Passing to the limit in the latter relation as $n \rightarrow \infty$ and then as $N \rightarrow 0$, we prove (25).

Since

$$
\sigma_{0}\left(\zeta_{n}(t)\right) \stackrel{\mathrm{P}}{\rightarrow} \sigma_{0}\left(\zeta_{0}(t)\right),
$$

$w_{n}(t)$ is a sequence of martingales, and $w_{n}(t) \stackrel{\mathrm{P}}{\rightarrow} w_{0}(t)$, we conclude that

$$
\int_{0}^{t} \sigma_{0}\left(\zeta_{n}(s)\right) d w_{n}(s) \stackrel{\mathrm{P}}{\rightarrow} \int_{0}^{t} \sigma_{0}\left(\zeta_{0}(s)\right) d w_{0}(s)
$$

(see [7]).

For the proof of convergence (26), we split the interval $[0,1]$ by the points $k h$. Taking into account Lemma 3.2 and well-known bounds for the stochastic Itô integrals we obtain

$$
\begin{aligned}
\mathrm{P}\left\{\sup _{0 \leq t \leq 1}\left|\int_{0}^{t} \sigma_{0}\left(\zeta_{n}(s)\right) d w_{n}(s)-\int_{0}^{t} \sigma_{0}\left(\zeta_{0}(s)\right) d w_{0}(s)\right|>\varepsilon\right\} \\
\leq \mathrm{P}\left\{\sup _{k}\left|\int_{0}^{k h} \sigma_{0}\left(\zeta_{n}(s)\right) d w_{n}(s)-\int_{0}^{k h} \sigma_{0}\left(\zeta_{0}(s)\right) d w_{0}(s)\right|>\frac{\varepsilon}{3}\right\} \\
+\mathrm{P}\left\{\sup _{k} \sup _{k h \leq t<(k+1) h}\left|\int_{k h}^{t} \sigma_{0}\left(\zeta_{n}(s)\right) \chi_{|x| \leq N}\left(\zeta_{n}(s)\right) d w_{n}(s)\right|>\frac{\varepsilon}{6}\right\} \\
+\mathrm{P}\left\{\sup _{k} \sup _{k h \leq t<(k+1) h}\left|\int_{k h}^{t} \sigma_{0}\left(\zeta_{0}(s)\right) \chi_{|x| \leq N}\left(\zeta_{0}(s)\right) d w_{0}(s)\right|>\frac{\varepsilon}{6}\right\} \\
+P_{N}+\mathrm{P}\left\{\sup _{0 \leq t \leq 1}\left|\zeta_{0}(t)\right|>N\right\}
\end{aligned}
$$




$$
\begin{aligned}
\leq & \sum_{k h<1} \mathrm{P}\left\{\left|\int_{0}^{k h} \sigma_{0}\left(\zeta_{n}(s)\right) d w_{n}(s)-\int_{0}^{k h} \sigma_{0}\left(\zeta_{0}(s)\right) d w_{0}(s)\right|>\frac{\varepsilon}{3}\right\} \\
& +\sum_{k h<1}\left(\frac{6}{\varepsilon}\right)^{4}\left(\frac{4}{3}\right)^{4} \mathrm{E}\left(\int_{k h}^{(k+1) h} \sigma_{0}\left(\zeta_{n}(s)\right) \chi_{|x| \leq N}\left(\zeta_{n}(s)\right) d w_{n}(s)\right)^{4} \\
& +\sum_{k h<1}\left(\frac{6}{\varepsilon}\right)^{4}\left(\frac{4}{3}\right)^{4} \mathrm{E}\left(\int_{k h}^{(k+1) h} \sigma_{0}\left(\zeta_{0}(s)\right) \chi_{|x| \leq N}\left(\zeta_{0}(s)\right) d w_{0}(s)\right)^{4} \\
& +P_{N}+\varlimsup_{n \rightarrow \infty} P_{N} \\
\leq & o(1)+\frac{C}{\varepsilon^{4}} \sup _{|x| \leq N} \sigma_{0}^{4}(x) \sum_{k h<1} h^{2}+P_{N}+\varlimsup_{n \rightarrow \infty} P_{N} .
\end{aligned}
$$

Passing to the limit as $n \rightarrow \infty$, then as $h \rightarrow 0$, and finally as $N \rightarrow \infty$, we prove (26). Equality (10) and relations (20) and (23)-(26) imply

$$
\sup _{0 \leq t \leq 1}\left|\zeta_{n}(t)-\zeta_{0}-\int_{0}^{t} a_{0}\left(\zeta_{0}(s)\right) d s-\int_{0}^{t} \sigma_{0}\left(\zeta_{0}(s)\right) d w_{0}(s)\right| \stackrel{\mathrm{P}}{\rightarrow} 0
$$

as $n \rightarrow \infty$. This means that there exists a subsequence $n^{\prime \prime}$ of the sequence $n^{\prime}$ for which convergence (27) is almost sure as $n^{\prime \prime} \rightarrow \infty$. Therefore

$$
F\left(\zeta_{n^{\prime \prime}}(t)\right) \rightarrow F\left(\zeta_{0}(t)\right)
$$

with probability one as $n^{\prime \prime} \rightarrow \infty$ for an arbitrary functional $F$ that is continuous in the space $C[0,1]$. Thus the process $\zeta_{n^{\prime \prime}}(t)$ weakly converges to $\zeta_{0}(t)$ (see [1]). Therefore the limit processes for the sequence of processes $\zeta_{n}(t)$ are solutions of equation $(22)$. Now Theorem 3.1 follows from the weak uniqueness of solutions of equation (22).

Corollary 3.1. If assumption 3) is excluded from the set of assumptions in Theorem 3.1, then the sequence of random polygonal lines $\zeta_{n}(t)$ is weakly compact and the limit points of this sequence are solutions of equation (22).

Theorem 3.2. Let all the assumptions of Lemma 3.4 hold. Assume that there exist functions $a_{0}(x)$ and $\sigma_{0}(x)$ that are continuous everywhere in the domain $|x| \leq N(N<$ $\infty$ is arbitrary) except for a finite set of points of discontinuity of the first kind. If assumptions 1)-3) of Theorem 3.1 hold, then the random polygonal line $\zeta_{n}(t)$ weakly converges as $n \rightarrow \infty$ to the solution $\zeta_{0}(t)$ of equation (22).

Proof. Similarly to the proof of Theorem 3.1, it is necessary to prove analogs of relations (23) -(26). Consider an arbitrary $N>0$. Let $x_{1}, x_{2}, \ldots, x_{m}$ be the points of discontinuity of the functions $a_{0}(x)$ and $\sigma_{0}(x)$ in the domain $|x| \leq N$. Let

$$
A^{(\varepsilon)}=\bigcup_{k=1}^{m}\left\{x:\left|x-x_{k}\right|<\varepsilon\right\} .
$$

Choose a sufficiently small number $\delta>0$ such that

$$
A^{(\delta)} \subset\{x:|x| \leq N\}
$$

and consider smooth versions of the functions $a_{0}(x)$ and $\sigma_{0}(x)$ on the subset $A^{(\delta)}$. Denote the smooth versions by $a^{(\delta)}(x)$ and $\sigma^{(\delta)}(x)$, respectively. 
It is clear that an analog of convergence (23) holds if $S_{n}^{(2)} \stackrel{\mathrm{P}}{\rightarrow} 0$ as $n \rightarrow \infty$. For all $\varepsilon>0$

$$
\begin{aligned}
\mathrm{P}\left\{S_{n}^{(2)}>\varepsilon\right\} \leq & P_{N}+\frac{2}{\varepsilon} \mathrm{E} \sum_{i=0}^{k_{n}-1} \int_{t_{n i}}^{t_{n i+1}}\left|a_{0}\left(\zeta_{n}(s)\right)-a_{0}\left(\zeta_{n}\left(t_{n i}\right)\right)\right| \chi_{|x| \leq N}\left(\zeta_{n}(s)\right) d s \\
\leq & P_{N}+\frac{2}{\varepsilon} \mathrm{E} \sum_{i=0}^{k_{n}-1} \int_{t_{n i}}^{t_{n i+1}}\left|a_{0}^{(\delta)}\left(\zeta_{n}(s)\right)-a_{0}^{(\delta)}\left(\zeta_{n}\left(t_{n i}\right)\right)\right| \chi_{|x| \leq N}\left(\zeta_{n}(s)\right) d s \\
& +\frac{2}{\varepsilon} \mathrm{E} \sum_{i=0}^{k_{n}-1} \int_{t_{n i}}^{t_{n i+1}}\left|a_{0}\left(\zeta_{n}(s)\right)-a_{0}^{(\delta)}\left(\zeta_{n}(s)\right)\right| \chi_{|x| \leq N}\left(\zeta_{n}(s)\right) d s \\
& +\frac{2}{\varepsilon} \mathrm{E} \sum_{i=0}^{k_{n}-1} \int_{t_{n i}}^{t_{n i+1}}\left|a_{0}\left(\zeta_{n}\left(t_{n i}\right)\right)-a_{0}^{(\delta)}\left(\zeta_{n}\left(t_{n i}\right)\right)\right| \chi_{|x| \leq N}\left(\zeta_{n}\left(t_{n i}\right)\right) d s \\
= & P_{N}+J_{n}^{(1)}+J_{n}^{(2)}+J_{n}^{(3)},
\end{aligned}
$$

where $J_{n}^{(k)}, 1 \leq k \leq 3$, denote the corresponding terms on the left hand side of the latter equality. Since the function $a_{0}^{(\delta)}(x)$ is continuous in the domain $|x| \leq N$, one can prove the convergence $J_{n}^{(1)} \stackrel{\mathrm{P}}{\rightarrow} 0$ as $n \rightarrow \infty$ by following the same line as that used in the proof of the convergence $S_{n}^{(2)} \stackrel{\mathrm{P}}{\rightarrow} 0$ in Theorem 3.1. Since

$$
J_{n}^{(3)} \leq \frac{2}{\varepsilon} C_{N} \sum_{i=0}^{k_{n}-1} \int_{t_{n i}}^{t_{n i+1}} \mathrm{P}\left\{\zeta_{n}\left(t_{n i}\right) \in A^{(\delta)}\right\} d s
$$

we derive from Lemma 3.4 that $J_{n}^{(3)} \stackrel{\mathrm{P}}{\rightarrow} 0$ by passing to the limit as $n \rightarrow \infty$ and then as $\delta \rightarrow 0$. Further,

$$
\begin{aligned}
J_{n}^{(2)} & =\frac{2}{\varepsilon} \mathrm{E} \sum_{i=0}^{k_{n}-1} \int_{t_{n i}}^{t_{n i+1}}\left|a_{0}\left(\zeta_{n}(s)\right)-a_{0}^{(\delta)}\left(\zeta_{n}(s)\right)\right| \chi_{|x| \leq N}\left(\zeta_{n}(s)\right) \chi_{A^{(\delta)}}\left(\zeta_{n}(s)\right) d s \\
& \leq \frac{2}{\varepsilon} C_{N} \int_{0}^{1} \mathrm{P}\left\{\zeta_{n}(s) \in A^{(\delta)}\right\} d s .
\end{aligned}
$$

In view of Lemma 3.5 and convergence (16), the right hand side of this inequality approaches zero if we let $n \rightarrow \infty$ and then $\delta \rightarrow 0$. Thus $J_{n}^{(2)} \stackrel{\mathrm{P}}{\rightarrow} 0$ as $n \rightarrow \infty$. Since $P_{N} \rightarrow 0$ as $N \rightarrow \infty$, we have $S_{n}^{(2)} \stackrel{\mathrm{P}}{\rightarrow} 0$ as $n \rightarrow \infty$.

The proof of an analog of convergence (24) is the same as above.

The only difference in the proof of an analog of (25) is that we consider a smooth version of $a_{0}(x)$ in the domain $|x| \leq N$ and use Lemma 3.4 to prove that

$$
\sup _{0 \leq t \leq 1}\left|\int_{0}^{1} a_{0}\left(\zeta_{n}(s)\right) q_{N}\left(\zeta_{n}(s)\right) d s-\int_{0}^{1} a_{0}\left(\zeta_{0}(s)\right) q_{N}\left(\zeta_{0}(s)\right) d s\right| \stackrel{\mathrm{P}}{\rightarrow} 0
$$

as $n \rightarrow \infty$.

The proof of convergence (26) is completely the same. The rest of the proof is the same as in the case of Theorem 3.1

\section{BIBLIOGRAPHY}

1. I. I. Gikhman and A. V. Skorokhod, Introduction to the Theory of Random Processes, Nauka, Moscow, 1965; English transl., W. B. Saunders Co., Philadelphia, 1969. MR.0247660 (40:923)

2. I. I. Gikhman and A. V. Skorokhod, Stochastic Differential Equations, Naukova Dumka, Kiev, 1968; English transl., Springer-Verlag, Berlin-Heidelberg-New York, 1972. MR0346904 (49:11625) 
3. N. V. Krylov, Controlled Diffusion Processes, Nauka, Moscow, 1977; English transl., SpringerVerlag, Berlin-New York, 1980. MR.508417 (80f:60046); MR0601776 (82a:60062)

4. G. L. Kulinich, Some limit theorems for a sequence of Markov chains, Teor. Veroyatnost. Matem. Statist. 12 (1975), 77-89; English transl. in Theory Probab. Math. Statist. 12 (1976), 79-92. MR0397835 (53:1691)

5. G. L. Kulinich and E. P. Kaskun, On the asymptotic behavior of solutions of one-dimensional Ito's stochastic differential equations with singularity points, Theory Stoch. Process. 4(20) (1998), no. 1-2, 189-197. MR2026628 (2004j:60122)

6. G. L. Kulinich, Necessary and sufficient conditions for the convergence of solutions of onedimensional stochastic differential equations with irregular dependence of the coefficients on a parameter, Teor. Veroyatnost. Primenen. 27 (1982), no. 4, 795-801; English transl. in Theory Probab. Appl. 27 (1982), no. 4, 856-862. MR681473 (84g:60093)

7. G. L. Kulinich, Asymptotic Analysis of Unstable Solutions of One-Dimensional Stochastic Differential Equations, Kyiv University, Kyiv, 2003. (Ukrainian)

8. A. V. Skorokhod, Studies in the Theory of Random Processes, Kiev University, Kiev, 1961; English transl., Scripta Technica, Inc. Addison-Wesley Publishing Co., Inc., Reading, Mass., 1965. MR0185620 (32:3082b)

Department of General Mathematics, Faculty for Mechanics and Mathematics, National Taras Shevchenko University, Academician Glushkov Avenue 6, Kyiv 03127, Ukraine

E-mail address: a_yershov@univ.kiev.ua

Department of General Mathematics, Faculty for Mechanics and Mathematics, National Taras Shevchenko University, Academician Glushkov Avenue 6, Kyiv 03127, Ukraine

Received 7/MAY/2007

Translated by S. KVASKO 